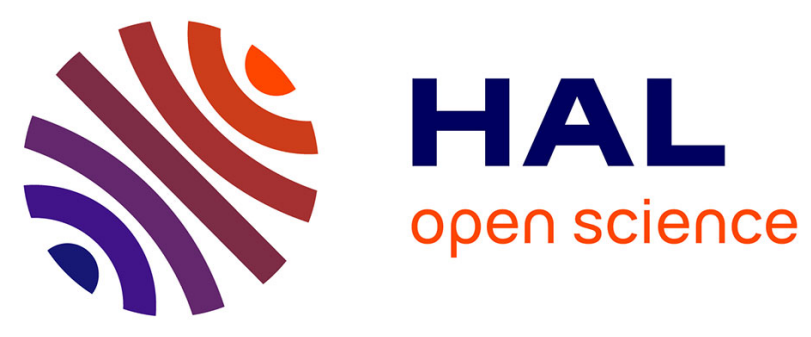

\title{
Filamentation and Pulse Self-compression in the Anomalous Dispersion Region of Glasses
}

\author{
A Couairon, V Jukna, J Darginavičius, D Majus, N Garejev, I Gražuleviči ${ }^{-}$ \\ Ut, G Valiulis, G Tamošauskas, A Dubietis, F. Silva, et al.
}

\section{To cite this version:}

A Couairon, V Jukna, J Darginavičius, D Majus, N Garejev, et al.. Filamentation and Pulse Selfcompression in the Anomalous Dispersion Region of Glasses. Andre D. Bandrauk; Emmanuel Lorin; Jerome V. Moloney. Laser Filamentation, Springer International Publishing, pp.147-165, 2016, CRM Series in Mathematical Physics, 10.1007/978-3-319-23084-9_6 . hal-01218982

\section{HAL Id: hal-01218982}

\section{https://hal-ensta-paris.archives-ouvertes.fr/hal-01218982}

Submitted on 21 Oct 2015

HAL is a multi-disciplinary open access archive for the deposit and dissemination of scientific research documents, whether they are published or not. The documents may come from teaching and research institutions in France or abroad, or from public or private research centers.
L'archive ouverte pluridisciplinaire HAL, est destinée au dépôt et à la diffusion de documents scientifiques de niveau recherche, publiés ou non, émanant des établissements d'enseignement et de recherche français ou étrangers, des laboratoires publics ou privés. 


\title{
Filamentation and pulse self-compression in the anomalous dispersion region of glasses
}

\author{
A. Couairon, V. Jukna, J. Darginavičius, D. Majus, N. Garejev, I. Gražulevičiūtè, \\ G. Valiulis, G. Tamošauskas, A. Dubietis, F. Silva, D.R. Austin, M. Hemmer, M. \\ Baudisch, A. Thai, J. Biegert, D. Faccio, A. Jarnac, A. Houard, Y. Liu, A. \\ Mysyrowicz, S. Grabielle, N. Forget, A. Durécu, M. Durand, K. Lim, E. McKee, \\ M. Baudelet and M. Richardson
}

\begin{abstract}
The propagation of near-infrared ultra-short laser pulses in the regime of anomalous dispersion of transparent solids is associated with a host of self-induced effects including a significant spectral broadening extending from the ultraviolet into the infrared region, pulse self-compression down to few-cycle pulse durations, free and driven third harmonic generation, conical emission and the formation of stable filaments over several $\mathrm{cm}$ showing the emergence of conical light bullets. We
\end{abstract}

A. Couairon, V. Jukna

Centre de Physique Théorique, CNRS, Ecole Polytechnique, F-91128, Palaiseau, France, e-mail: couairon@cpht.polytechnique.fr

J. Darginavičius, D. Majus, N. Garejev, I. Gražulevičiūtè, G. Valiulis, G. Tamošauskas, A. Dubietis

Department of Quantum Electronics, Vilnius University, Sauletekio Avenue 9, Building 3, LT10222 Vilnius, Lithuania,

F. Silva, D.R. Austin, M. Hemmer, M. Baudisch, A. Thai, J. Biegert

ICFO Institut de Ciencies Fotoniques, Mediterranean Technology Park, 08860 Castelldefels (Barcelona), Spain, and ICREA - Instituci Catalana de Recerca i Estudis Avanats, 08010 Barcelona, Spain

D. Faccio

School of Engineering and Physical Sciences, Heriot-Watt University, Edinburgh EH14-4AS, United Kingdom

M. Durand, A. Jarnac, A. Houard, Y. Liu, A. Mysyrowicz

Laboratoire dOptique Appliquée, ENSTA ParisTech, Ecole Polytechnique, CNRS, F-91762

Palaiseau, France

S. Grabielle, N. Forget

FASTLITE, Centre Scientifique dOrsay, Plateau du Moulon, F-91401 Orsay, France,

A. Durécu,

Onera-The French Aerospace Lab, BP 80100, F-91123 Palaiseau cedex, France

M. Durand, K. Lim, E. McKee, M. Baudelet and M. Richardson

Townes Laser Institute, CREOL-The College of Optics and Photonics, University of Central Florida, Orlando, Florida 32816, USA 
review measurements performed in different experimental conditions and results of numerical simulations of unidirectional propagation models showing that the interpretation of all these phenomena proceed from the formation of non-spreading conical light bullets during filamentation.

\section{Introduction}

Laser pulses with central wavelengths in the near and mid-infrared region are available in many laboratories. All sources are exclusively based on optical parametric amplifiers since there are no femtosecond lasers operating in this range. As shown in Figure 1, which illustrates the variation of the second order dispersion coefficient $k_{0}^{\prime \prime}$ as a function of wavelength for different materials, near- and mid-infrared laser sources operating at $2 \mu \mathrm{m}$ or $3 \mu \mathrm{m}$ lie within the region of anomalous dispersion for fused silica, calcium fluoride and YAG.

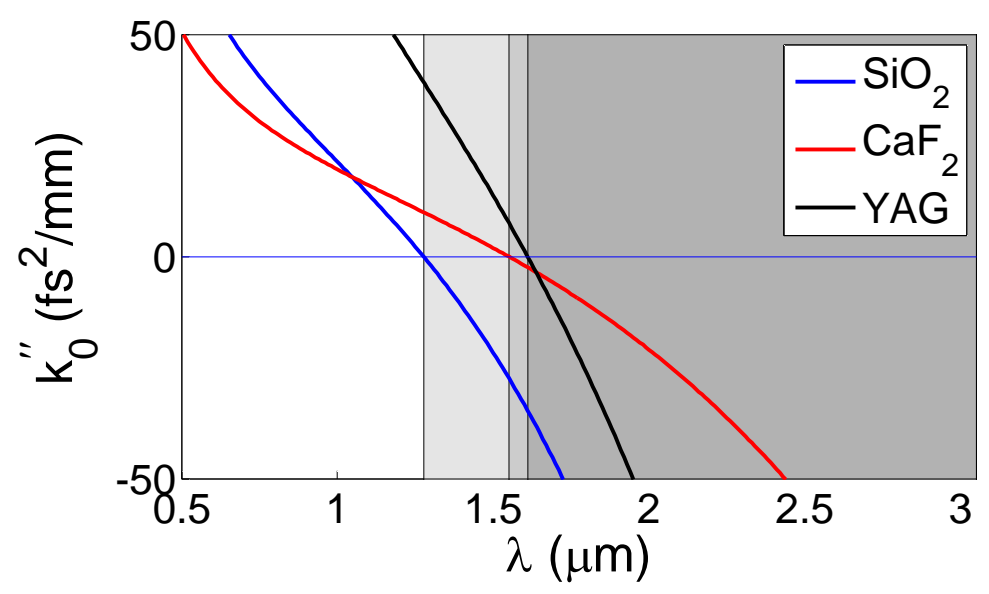

Fig. 1 Group velocity dispersion of fused silica, CaF2 [30] and YAG [1]. For each material, the gray area indicate the anomalous dispersion region.

This opens up new possibilities for filament based applications [4]: for instance, nonlinear pulse propagation in this regime leads to pulse compression rather than pulse splitting and the possibility for ultrabroadband generation from supercontinuum generation. The generation of odd harmonics in the lowest orders falls into the visible region providing a direct probing of higher order nonlinearities. The development of near and mid-infrared laser sources also led to new investigations on the fundamentals of filamentation in transparent solids. Several publications in this area during the last last two years $[27,15,5,6,7,13,20,14]$ uncovered filamentation physics specific to the anomalous dispersion regime and clarified the links between pioneering results $[21,18,25]$. 
In 2003, Moll and Gaeta investigated the role of dispersion in multiple collapse dynamics [21]. On the basis of experiments and numerical simulations, they have shown that the filamentation legth is extended in the anomalous dispersion regime: collapse events can occur at locations in the medium many diffraction lengths beyond the initial collapse point, in contrast with the normal dispersion regime in which multiple collapse occurs within a diffraction length.

In 2005, Kolesik, Wright and Moloney proposed an interpretation of conical emission of femtosecond laser pulses propagating in bulk media [18], valid in the normal as well as in the anomalous dispersion regime. They have shown that the input pulse with initially localized spectrally resolved far-field, is scattered off a material wave, which is nothing but the self generated nonlinear polarization wave associated with nonlinear propagation of the pulse. This results in preferential locations for populating the far-field, which can be predicted by an effective three wave mixing model and phase matching arguments.

The same year, Porras et al. have discovered a new type of spatiotemporal light bullet, which is nonsolitary and exhibit a conical nature [25]. Concretely, they have found a stationary solution to an extended nonlinear Schrödinger equation describing propagation in a Kerr medium, in the regime of anomalous dispersion, and in the presence of nonlinear losses. This solution represents a nonlinear and stable statio-temporal wavepacket that does not spread in space or time and withstands nonlinear losses. For this reason, it differs from a soliton or a light bullet as defined by Silberberg [26]. Figure 2 shows a typical profile for this solution, which takes the form of a nonlinear Bessel function along a reduced coordinate $R \equiv \sqrt{r^{2}+t^{2} / k_{0} k_{0}^{\prime \prime}}$ combining the transverse spacial coordinate $r$ and the pulse local time $t$, where $k_{0}$ and $k_{0}^{\prime \prime}$ denote the wavenumber and the second order dispersion coefficient in the medium at the central wavelength of the pulse. These solutions have a ring shaped spectrum indicating that wavenumbers are distributed along the surface of a cone. This is typical for Bessel-like profiles. This solution can therefore be viewed as a nonlinear polychromatic Bessel beam, i.e., a light bullet with a conical nature.

The goal of this chapter is to show the links between these three results in light of recent experimental results which led us to discover a new filamentation regime, featured by pulse self-compression, stationarity and the formation of similar light bullets as those predicted by Porras et al. [25]. Figure 3 illustrates this new filamentation regime, obtained by recording with a CCD camera the side image of the blue luminescence after focusing the beam of a $40 \mathrm{fs}, 8 \mu \mathrm{J}$ laser pulse at wavelength of $1.9 \mu \mathrm{m}$ on the entrance surface of a $3 \mathrm{~cm}$-thick block of fused silica. The measurement reveals a thin and regular filamentary track extending over a large part of the sample in the anomalous dispersion regime, whereas a significantly shorter filament with multiple refocusing cycles is obtained in the regime of normal dispersion [6].

The outline of this chapter is the following. Section 2 briefly presents the model used in this work for numerical simulations of nonlinear pulse propagation and filamentation. Section 3 deals with investigations on ultrabraodbband supercontinuum generation. Section 4 shows that these spectra are a signature of efficient pulse selfcompression. Section 5 shows that a visible spectral peak appears in the ultrabroadband spectra, corresponding to the axial part of conical emission. Section 6 delas 

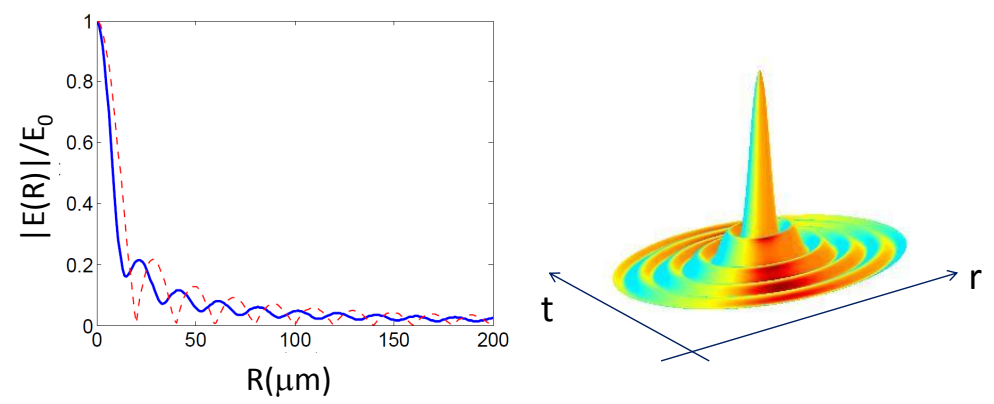

Fig. 2 (a) Amplitude profile for conical light bullets (nonlinear O-waves from the theory in Ref. [25]) supported by anomalous dispersion with normalized intensity. Solutions exist as a continuous family parametrized by the maximum amplitude. In this example, the dashed red curve corresponds to a linear solution. The continuous blue curve is typical for nonlinear solutions and corresponds to the a peak intensity of $9 \times 10^{12} \mathrm{~W} / \mathrm{cm}^{2}$ (b) Space-time representation of the ampliude of conical light bullets.

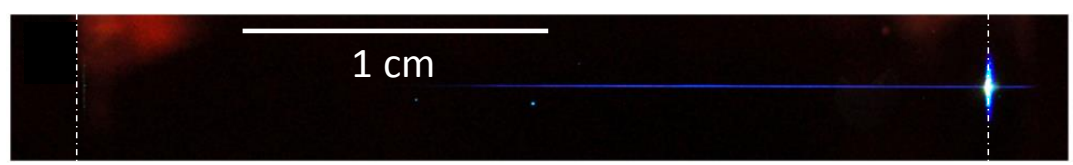

Fig. 3 Side view photography of the blue fluorescence of fused silica induced by a near infrared laser pulse at $1.9 \mu \mathrm{m}$ with $8 \mu \mathrm{J}$ energy. The laser pulse is focused on a $70 \mu \mathrm{m}$ spot (FWHM) at the entrance surface of the $3 \mathrm{~cm}$ thick sample. Vertical dash-dotted lines indicate the positions of the entrance and exit surfaces of the sample. Adapted from [6].

with free and driven third harmonic generation and finally section 7 shows measurements and simulations on a new filamentation regime where dissipative spatiotemporal light bullets emerge.

\section{Numerical simulation Model}

For interpreting the measurements, numerical simulations were performed on the basis of unidirectional propagation equations [16, 3]. The Maxwell Wave Equation (MWE) describes the evolution of the pulse electric field along $z$, the propagation axis.

$$
\frac{\partial \hat{E}(z, \omega, r)}{\partial z}=i\left(k(\omega)-k_{0}^{\prime} \omega\right) \hat{E}+\frac{i}{2 k(\omega)} \nabla_{\perp}^{2} \hat{E}+\frac{1}{2 \varepsilon_{0} c n(\omega)}[i \omega \hat{P}(z, \omega, r)-\hat{J}(z, \omega, r)]
$$

where $\hat{E}(z, \omega, r)=\mathscr{F}[E(z, t, r)]$ denotes the frequency components (Fourier transform $t \rightarrow \omega)$ of the laser electric field $E(z, t, r)$ and the transverse coordinate $r$ indicates that revolution symmetry is assumed. The effects accounted for include mate- 
rial dispersion via a Sellmeier dispersion relation for the medium $k(\omega)$, diffraction, and the nonlinear response of the medium described by the nonlinear polarization

$$
P(z, t, r) \equiv \varepsilon_{0} \chi^{(3)} E^{3}(z, t, r)
$$

and current $J(z, t, r)$ source terms. These terms in turn represent the main effects playing a role in filamentation: the optical Kerr effect with nonlinear index coefficient $n_{2},\left(\chi^{(3)} \equiv(4 / 3) \varepsilon_{0} c n_{0}^{2} n_{2}\right)$, plasma effects including ionization, plasma defocusing and absorption and nonlinear absorption governed by the system of equations $(3,4,5)$

$$
\begin{array}{r}
\frac{\partial J_{e}}{\partial t}+\frac{J_{e}}{\tau_{c}}=\frac{q_{e}^{2}}{m_{e}} \rho E(z, t, r) \\
\frac{\partial \rho}{\partial t}=W(E)\left(1-\rho / \rho_{b}\right)+\frac{\sigma}{U_{i}} \rho E^{2} \\
J(z, t, r)=J_{e}+\varepsilon_{0} c n_{0} \frac{W(E)}{E^{2}} U_{i}\left(1-\rho / \rho_{b}\right) E,
\end{array}
$$

where $q_{e}$ and $m_{e}$ denote the electron charge and mass, $\tau_{c}$ the effective collision time, $\sigma$ the cross section for inverse Bremsstrahlung, $U_{i}$ the bandgap of the material and $\rho_{b}$ the background neutral atom density. Optical field ionization rates $W(E)$ are described by the Keldysh formulation. The model is valid for describing the propagation of few-cycle pulses with broad spectra. A formally analogous formulation based on an envelope propagation equation will be also used when explicitely specified. In the latter case, ionization rates and nonlinearity depend on intensity rather than on the field squared.

\section{Supercontinuum generation}

\subsection{Supercontinuum generation in glasses at $2 \mu \mathrm{m}$}

Figure 4 shows a series of measurements for the supercontinuum spectra obtained by propagation of $15 \mathrm{fs}$ ( 2.3 optical-cycle), CEP-stable pulses with central wavelength of $2 \mu \mathrm{m}$ in different solid media, fused silica, $\mathrm{CaF}_{2}$ and YAG. The corresponding conical emission is shown as a cross section of the emerging beam taken at $15 \mathrm{~cm}$ distance from the output face of the nonlinear medium.

Different features are observed: (i) generation of supercontinuum spectra; (ii) presence of a spectral peak in the visible region; (iii) emergence of a third harmonic peak for moderate pulse energy and (iv) conical emission in the form of angular spreading of visible light. These features are quite generic and reproducible in different media, at other pulse wavelengths and durations.

In fused silica, the supercontinuum spectrum has a deep extended minimum around $1 \mu \mathrm{m}$ and a distinct intense peak in the visible (blue peak), which shows 

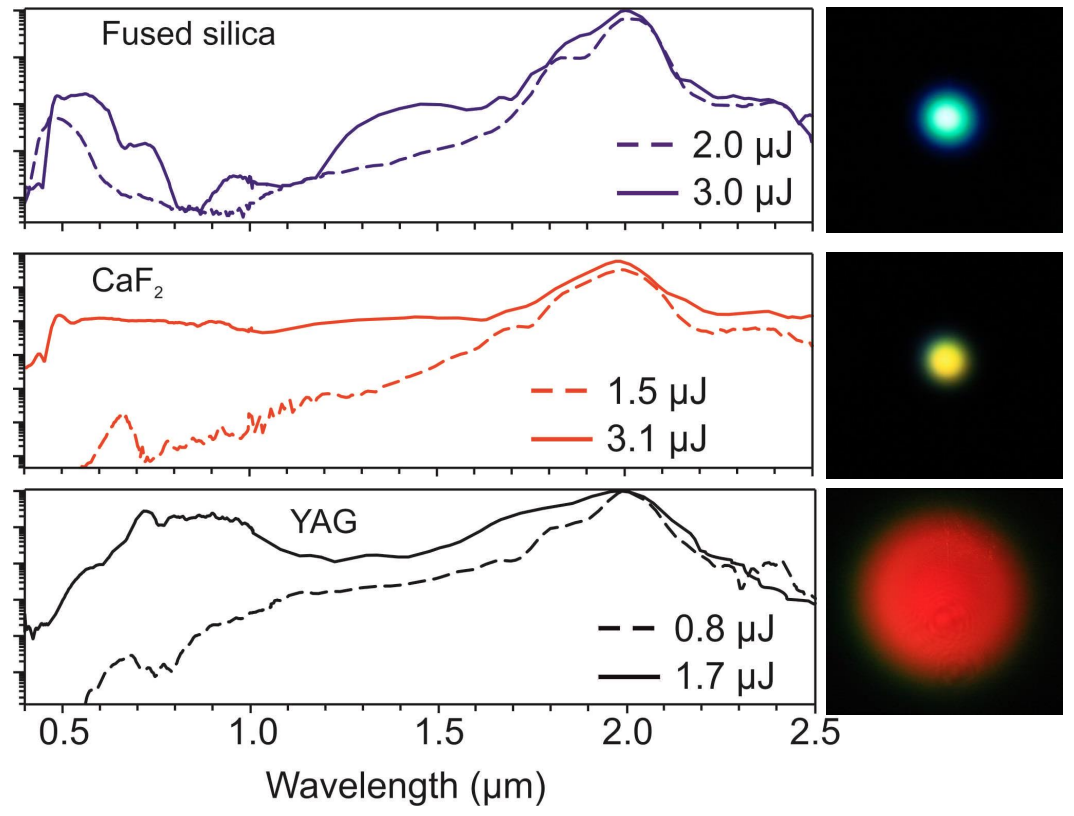

Fig. 4 Supercontinuum spectra generated in (a) fused silica, (b) CaF2, (c) YAG. The dashed and solid curves represent Supercontinuum spectra in the transient and saturation regimes of the spectral broadening, respectively. Curve labels stand for the input-pulse energy. Images on the right side show the corresponding far-field patterns of the conical emission in the visible range, recorded in the saturated regime of the spectral broadening. Adapted from [5].

red-shifted broadening with increase of the input-pulse energy. $\mathrm{CaF}_{2}$ exhibits lower dispersion than fused silica: a broad and flat supercontinuum spectrum from 450 $\mathrm{nm}$ to more than $2.5 \mu \mathrm{m}$ was generated. In YAG which has higher nonlinearity, a smooth supercontinuum spectrum with elevated spectral intensity in the visible near-infrared 600-1000 $\mathrm{nm}$ range was generated using low input-pulse energy. The differences in conical emission bear upon the characteristic coloring and angular divergence of the supercontinuum radiation in the saturated regime of the spectral broadening, corresponding to higher values of the input-pulse energy. Among the tested materials YAG has the highest nonlinear index of refraction, leading to a strong laser-matter interaction, and the largest dispersion, therefore visible extension of supercontinuum in YAG has the largest angular spread. This feature will be further commented in section 5 .

In all cases, prior to supercontinuum generation, a characteristic third harmonic peak centered at $660 \mathrm{~nm}$ was observed. Its nature is discussed in section 6. It is best visible in $\mathrm{CaF}_{2}$ and YAG. In fused silica, it becomes rapidly masked by the strong and broad visible peak when energy is increased, which is distinct froml the third harmonic as will be thoroughly explained in section 5 . 


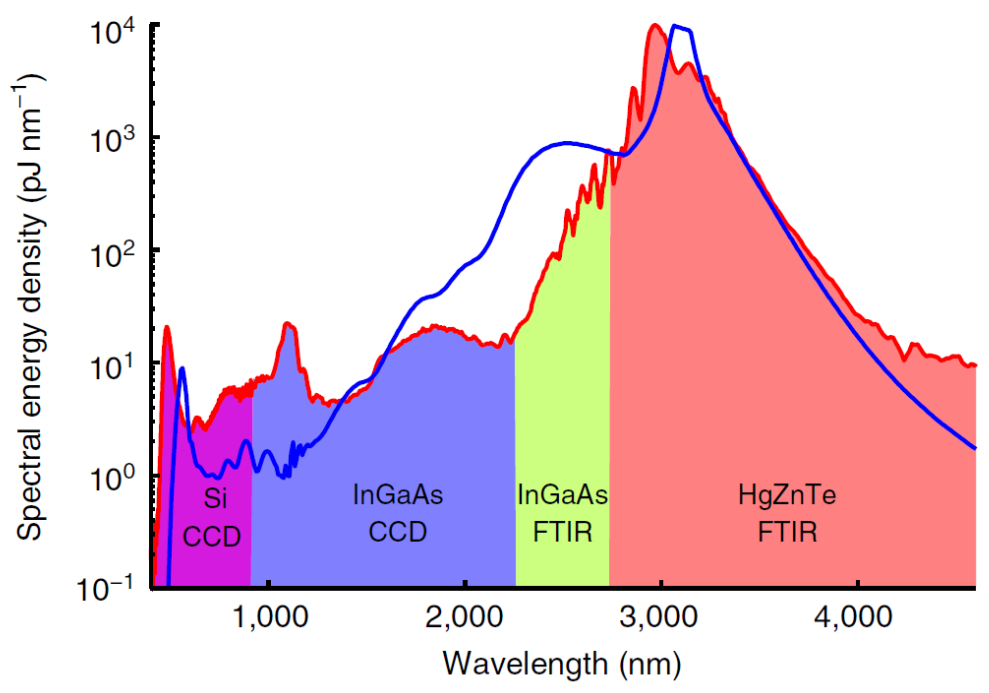

Fig. 5 Supercontinuum generated by $3100 \mathrm{~nm}, 2.6 \mu \mathrm{J}$ pulses in YAG (red). The ranges of each spectrometer/detector are indicated and superimposed is the angle-integrated spectrum from our simulation (blue). Two different detectors (InGaAs and $\mathrm{HgCdZnTe}$ ) were used to cover the long wavelength region. FTIR, Fourier Transform Infrared Spectrometer. Adapted from [27].

\subsection{Supercontinuum generation in YAG at $3 \mu \mathrm{m}$}

Recent experimental results have shown ultrabroadband supercontinuum generation in a YAG cristal from mid-IR laser pulses with central wavelength of $3 \mu \mathrm{m}$ [27]. Pulses of $7 \mu \mathrm{J}$ energy were focused in a 2-mm-thick YAG plate placed in the focal region. It produced a clearly visible filament. After the exit plane of the YAG plate, we recorded spectra by imaging the supercontinuum into four spectrometer fibers. Each fiber delivered light to four different spectrometers working in different spectral regions, as indicated by the colored regions in 5 . Intensity calibrations were applied for ensuring consistency in their regions of overlap (925 2300 and $2700 \mathrm{~nm}$ ) and with the measured pulse energy.

The result is shown in Fig. 5, along with a corresponding numerical simulation. The spectrum shows that the long wavelength edge decays smoothly, reaching the noise level at $4.5 \mu \mathrm{m}$. The short wavelengths extend down to $450 \mathrm{~nm}$. The highest spectral energy density is found around the mid-infrared pump wavelength. The smallest energy content is observed in the $750-1000 \mathrm{~nm}$ range at a few picojoules per nanometer.

We observed two spectral peaks at $600 \mathrm{~nm}$ and $1100 \mathrm{~nm}$ seemingly close to the third and fifth harmonics. The blue curve corresponds to the result of a numerical simulation. It does reproduce well the peak in the visible region but not the third harmonic which merges in the supercontinuum. Another simulation with an envelope formulation, which does not account for harmonic generation, produced the same 


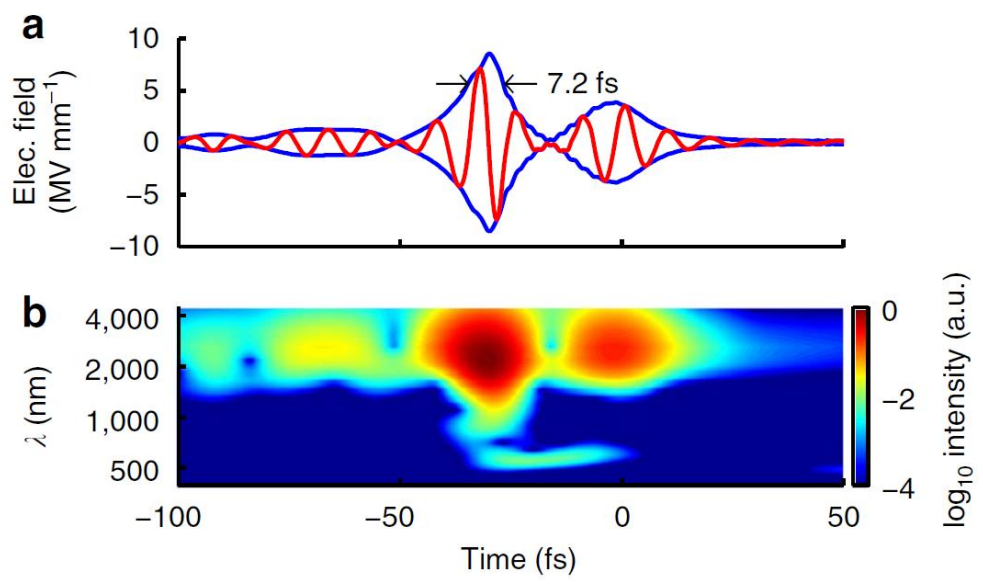

Fig. 6 Temporal profile and spectrogram of the output pulse after filamentation in YAG at $3 \mu \mathrm{m}$. (a) Temporal envelope (blue) and field oscillations (red) and (b) spectrogram of the on-axis simulated pulse emerging from the YAG plate; note the logarithmic intensity colour scale in the spectrogram. Adapted from [27].

features in the supercontinuum spectrum, in particular a prominent visible peak, suggesting that this peak at $600 \mathrm{~nm}$ is not related to fifth harmonic generation.

Simulations with the Maxwell wave equation 1 and 3-5 detailed how the spectrally resolved far-field is populated along propagation [27]. The visible peak appeared in the last $400 \mu \mathrm{m}$ of the YAG plate and is not affected by harmonic generation. Its generation is due to a scattering process explained in section5. It coincides with the loci of phase-matched angle and wavelength liying in the visible region of the spectrum, i.e., this peak is nothing but the axial part of conical emission. The presence of this spectral peak is also a strong signature of the partial reshaping of the pulse into O- or fishlike waves in media with anomalous dispersion [23, 8, 24].

We used the numerics to examine the potential of our setup for pulse compression. Figure 6a shows a typical output temporal on-axis profile obtained by numerical simulation. We found that the pulse duration has been reduced by a factor of 10 (the satellite peaks are $50 \%$ lower in peak intensity), and the main peak has nearsingle-cycle duration. A more complete picture of the output pulse is given by the spectrogram shown in Fig. 6b. The short wavelength components, below $1500 \mathrm{~nm}$, emerge from the output face nearly simultaneously with the main peak. The overall time-frequency distribution is simple, containing only a few distinct peaks. Therefore, the numerics indicate the possibility of achieving in an extremely simple setting, near-single-cycle and carrier-envelope phase stable pulses using mid-infrared pump lasers. 


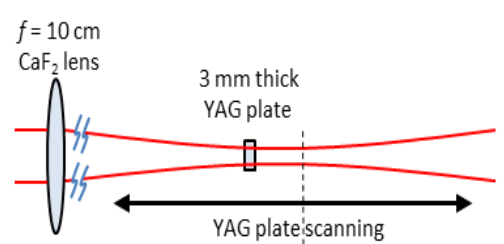

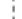

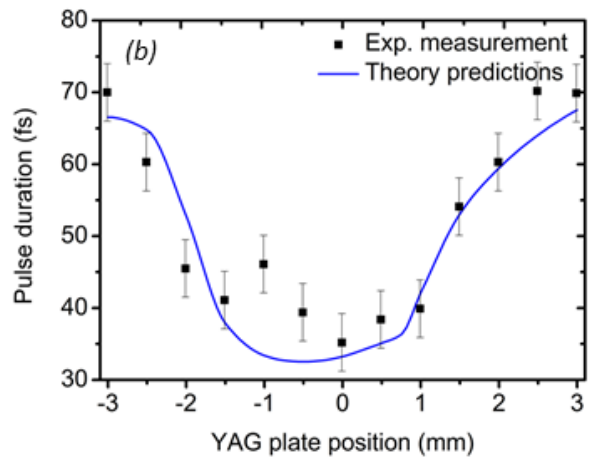

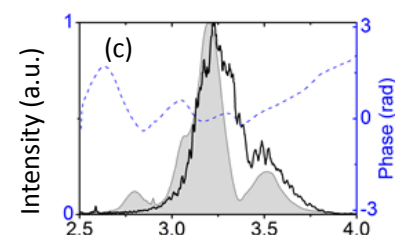
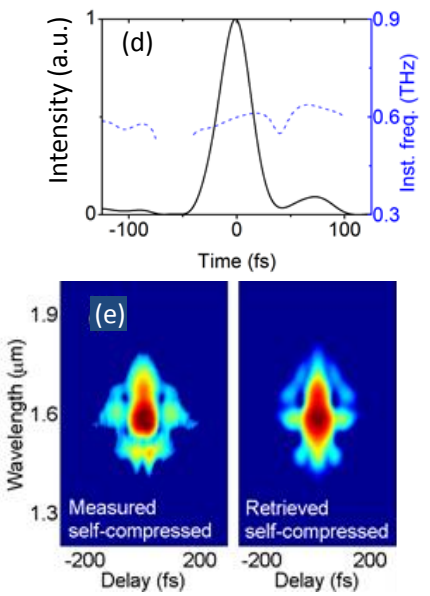

Fig. 7 (a) Layout of the self-compression setup. The input beam is focused by a $10 \mathrm{~cm}$ focal length $\mathrm{CaF} 2$ lens into a $3 \mathrm{~mm}$-thick YAG plate. The YAG plate is moved along the beam propagation axis, through focus. (b) measured (black dots) and simulated (blue line) evolution of the pulse duration as a function of YAG plate position through focus. (c) Measured (black line) and retrieved (shadow) spectra of the self-compressed pulses. (d) Retrieved normalized $32 \mathrm{fs}$ (2.9-cycle) duration intensity profile of the self-compressed pulses. (e) Measured and retrieved SHG-FROG traces of the selfcompressed pulses on logarithmic color scale. Adapted from [15].

\section{Pulse self-compression in the regime of anomalous dispersion in glasses}

We used the simple experimental setup sketched in Fig. 7a to investigate direct pulse self-compression. The parameters of this setup consist of a $10 \mathrm{~cm}$ focal length $\mathrm{CaF} 2$ lens and a 3-mm thick, Yttrium Aluminum garnet (YAG) plate which was moved 6 $\mathrm{mm}$ across the focal plane in steps of $0.5 \mathrm{~mm}$ to initiate nonlinear propagation inside the plate at different peak intensities. The emerging beam was collimated with a silver-coated mirror and the pulse was characterized with frequency-resolved optical gating (FROG). The FROG device was specifically designed to handle ultrabroad bandwidths in the mid-IR region [2]. Mid-IR spectra were also measured using a Fourier transform infrared spectrometer.

The results in Fig. 7c show measured and simulated durations of the emerging pulse as a function of the YAG plate position, representing the distance between the front of the YAG plate and the linear focus. Simulations and experiments are in good agreement. They indicate that self-compression to $32 \mathrm{fs}$ ( 2.9 cycle) occurs and that 
no pulse splitting is observed. Optimum self-compression conditions for $3 \mu \mathrm{J}$ seed pulses were identified and the optimally self-compressed pulses were carefully characterized by sampling the inner part of the beam. Figures $7 \mathrm{c}$,d,e show measured and retrieved FROG traces with a maximum $0.5 \%$ error. Figures $7 \mathrm{~d}$ shows the optimally self-compressed pulse. The measured spectrum in Fig. 7c for the optimally selfcompressed pulse shows an extent of over $1000 \mathrm{~nm}$ and the retrieved spectrum from the FROG measurement is in good agreement with the measured spectrum confirming the consistency of the measurement. We retrieve a FWHM pulse duration of 32 fs which corresponds to 2.9-cycles of electric field at the slightly red-shifted output of $3200 \mathrm{~nm}$. The spatial profile of the beam after self-compression was measured using a knife-edge technique and showed that the process left the seed spatial profile undisturbed.

\section{Conical emission}

Nonlinear propagation of an intense laser pulse generates new frequencies leading to the supercontinuum. The angular spread in the visible wavelength range is regarded as conical emission, whose angles are set by dispersion-related phase matching conditions. In other words, for a given material, the phase matching condition entirely determines the loci of the far-field that can be populated in priority, i.e., the dependence of angles as a function of wavelength in the conical emission pattern. The effective scattering of a specific color at a given angle also depends on the nonlinear pulse-matter interaction ensuring that the phase matching condition is fulfilled over a certain propagation distance.

As shown in Figs. 4 and 5, the third harmonic does not always emerge from the supercontinuum and cannot always be distinguished from the spectral peak in the visible region. For instance near-infrared pump pulses with central wavelength close to $2 \mu \mathrm{m}$ usually undergo spectral broadening and frequency shift during nonlinear propagation leading to a loosely defined third harmonic wavelength in the visible range.

Figure 8 (right column) indicates the physical origin of the visible spectral peak. We compared the spectrally resolved far-fields obtained by numerical simulation of filamentation in fused silica for different pulse wavelengths $\left(\lambda_{0}=800 \mathrm{~nm}, 1200 \mathrm{~nm}\right.$, $1900 \mathrm{~nm}$, see Fig. 8). We found that the generation of the visible peak is consistent with the effective three wave mixing model proposed by Kolesik et al. [18]. Due to the reshaping of the pulse during nonlinear propagation, the peak field and therefore the nonlinear polarization wave induced by the pulse (Eqs. 2-5) travels at velocity $v_{p}$, usually slightly lower than the pulse group velocity in the conditions of anomalous dispersion $[18,7]$. This constitutes a material wave, the velocity of which can be quantified from the results of numerical simulations by monitoring the temporal profile of the pulse along propagation (see example below in Fig. 12). Conical emission is essentially interpreted as a scattering process: the pump pulse is scattered off the material wave. Newly generated frequencies will populate the spectrum in re- 

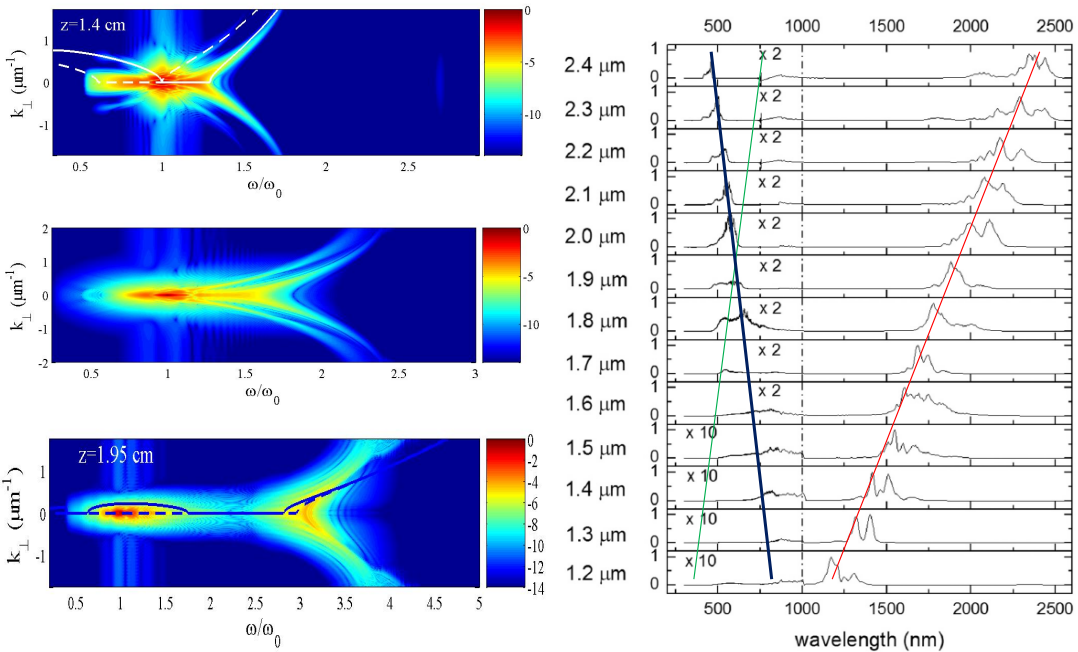

Fig. 8 Left column: Spectrally resolved far-fields $k_{\perp}-\omega$ obtained by numerical simulation of filamentation of $50 \mathrm{fs}$ laser pulses in fused silica, for different pump wavelengths: First row: $\lambda_{0}=$ $800 \mathrm{~nm}$ (normal dispersion). Second row: $\lambda_{0}=1200 \mathrm{~nm}$ (close to zero dispersion): Third row: $\lambda_{0}=1900 \mathrm{~nm}$ (anomalous dispersion). Units on the logarithmic colorbars corresponds to decades in intensity. Right column: Supercontinuum spectra measured after filamentation in a $4 \mathrm{~cm}$ thick fused silica sample, with variable pump central wavelengths from 1.2 to $2.4 \mu \mathrm{m}$. For legibility, magnification factors were applied in the region of the spectra up to $1 \mu \mathrm{m}\left(10 \times\right.$ for $1.2<\lambda_{0}<1.5$ $\mu \mathrm{m})$ and $2 \times$ for $\left.1.6<\lambda_{0}<2.4 \mu \mathrm{m}\right)$. Adapted from [7].

gions where the longitudinal phase matching condition (6) between the pump pulse (axial wavenumber $k_{0}$ ), the material wave (axial wavenumber $\left(\omega-\omega_{0}\right) / v_{p}$ ) and the scattered wave $\left(k_{z}\right)$ is fulfilled.

$$
\begin{array}{r}
\Delta k \equiv k_{0}+\frac{\omega-\omega_{0}}{v p}-k_{z}\left(\omega, k_{\perp}\right) \simeq 0, \\
k_{\perp}^{2}+k_{z}^{2}=k^{2}(\omega) .
\end{array}
$$

Equation (7) links transverse and longitudinal components for dispersive waves in the medium, and $k(\omega)$ denotes a Sellmeier like dispersion relation characterizing the medium. The angular distribution of frequencies (or wavelengths) where the farfield is preferentially populated along propagation is obtained by the combination of Eqs (6) and (7):

$$
k_{\perp}= \pm \sqrt{k^{2}(\omega)-\left[k_{0}-\Delta k+\alpha\left(\omega-\omega_{0}\right)\right]^{2}}
$$

where $\alpha \equiv v_{p}^{-1}$ and $\Delta k$ is a free parameter (zero for perfect phase matching).

In Fig. 8, we plotted the phase matching condition (8) for both cases of normal and anomalous dispersion. For $\lambda_{0}=800 \mathrm{~nm}$, we used two different values of the $\alpha$ parameter, corresponding to the two velocities of the split pulses arising in filaments 
with normal dispersion $[17,11]$. This leads to two pairs of hyperbolic branches (one pair for each velocity) highlighted by the white continuous and dashed curves). For $\lambda_{0}=1900 \mathrm{~nm}$, the same procedure leads to two branches: one is elliptic around the pump, a second one is hyperbolic and marks the conical emission in the visible range. Note that measurements of the spectrally resolved far-field are very well fitted by these branches both in the normal and the anomalous dispersion regimes [11, 18, $10,9,12]$, which confirms the physical origin of conical emission and highlights the nature of the spectral peak in visible region of the supercontinuum.

To further confirm that spectral peaks in the visible region originate from dispersive waves populating the supercontinuum and distinguish them from third harmonic generation, another set of experiments was performed on filamentation in fused silica, where the pulse wavelength was varied from 1.2 to $2.4 \mu \mathrm{m}$ [7]. The experiments clearly confirmed that the visible spectral peak is not related to the third harmonic but corresponds to the axial part of conical emission. The laser source was an OPA pumped by a titanium:sapphire chirped-pulse amplified laser system. The pulses at the output of the OPA had energies up to $100 \mu \mathrm{J}$, at $100 \mathrm{fs}$. The laser beam was focused with a $50 \mathrm{~cm}$ focal length lens inside a $3-\mathrm{cm}$-long fused silica hexagonal rod. The measurements taken by three spectrometers were combined to cover the spectral range from 300 to $2500 \mathrm{~nm}$. Results are shown in the right column of Fig. 8.

The visible spectral peak, highlighted by a blue line, is clearly shifted to shorter wavelengths when the initial pulse wavelength (red line) is varied to longer wavelengths, ruling out third-harmonic generation as the mechanism at the origin of the visible emission. The green line marks the position of the third harmonic, showing clearly that it is well separated from the visible peak except for a short range of pump wavelengths around $2 \mu \mathrm{m}$.

We have finally applied this model to determine the position of the visible peak as a function of the input pulse wavelengths. The results plotted in Fig. 9. The comparison of the model prediction, in solid black curve, with the measurements in red dots, shows very good agreement in the range of pump wavelengths $1500-2000 \mathrm{~nm}$ and a slight redshift with respect to the prediction in the range $2000-2400 \mathrm{~nm}$. The figure also shows the extent of the visible spectral peak.

\section{Third harmonic generation}

As shown in Fig. 4, third harmonic generation is clearly observed before the onset of spectral broadening and supercontinuum generation, in the transient regime of supercontinuum generation [5, 13]. Typically, the third harmonic radiation was detected with half the lowest pulse energies indicated in Fig. 4 in fused silica and in YAG, and the measured third harmonic efficiency varied from $10^{-6}$ to $10^{-4}$, depending on the input-pulse energy.

A closer inspection of third harmonic spectra revealed a fast periodic modulation, whose frequency changed with the nonlinear material and its length. An example of 
Fig. 9 Comparison of the wavelength of the spectral peak in the visible region obtained from the theory $\left(\lambda_{+, \text {theory }}\right.$, black curve) with the measurements $\left(\lambda_{+, \text {experiment }}\right.$, red dotted curve). The visible peak is generated during filamentation in a 3 -cm-thick fused silica sample, as a function of the input pulse central wavelength. The curves $\lambda_{-, \text {HWHM }}$ and $\lambda_{+, \text {HWHM mark the extent }}$ of the visible peak. Adapted from [7].

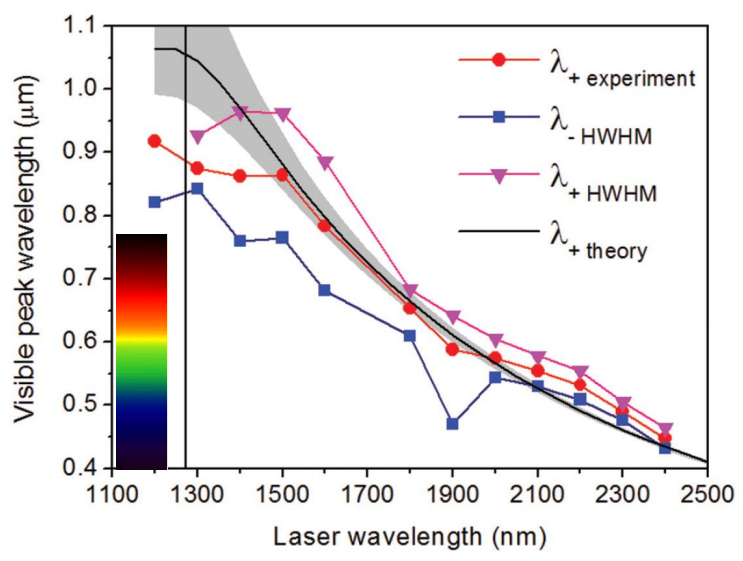

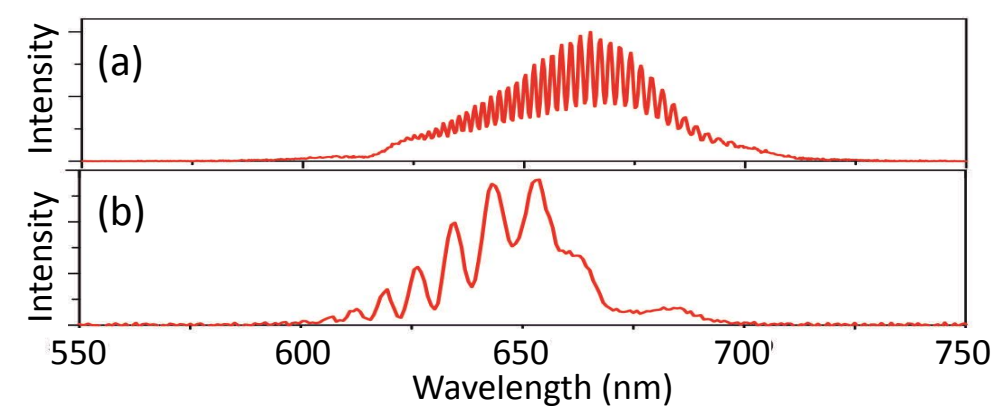
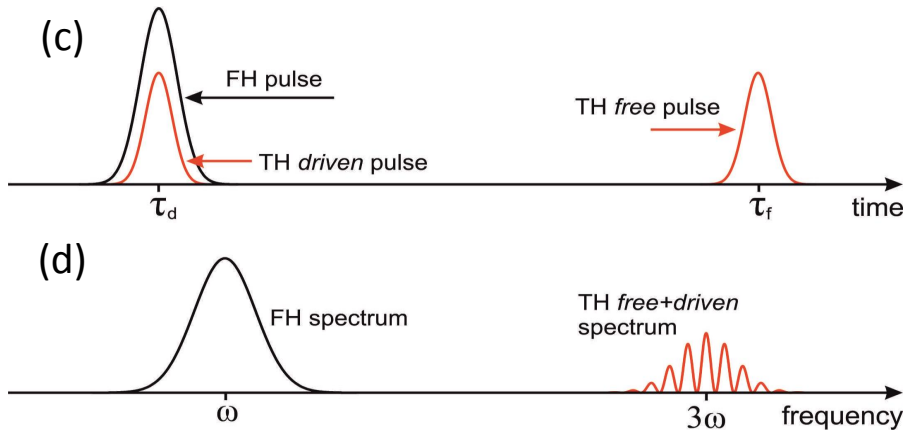

Fig. $10(a, b)$ Fringe pattern in the third harmonic spectra after propagation of a 15 fs pulse in (a) 6 $\mathrm{mm}$ of YAG, suggesting two pulses separated by $670 \mathrm{fs}$ and (b) $5 \mathrm{~mm}$ of fused silica. (c,d) Sketch for the generation of third harmonic radiation in the conditions of large phase and group velocity mismatch. (c) time domain and (d) frequency domain.

third harmonic spectrum generated with a 15 fs input-pulse with $0.70 \mathrm{~mJ}$ energy, before the onset of supercontinuum generation, is shown in Fig. 10(a,b) for YAG and fused silica, respectively. This spectral modulation is a signature of a double-peaked third harmonic pulse, which occurs naturally, without the splitting of the input pulse. 

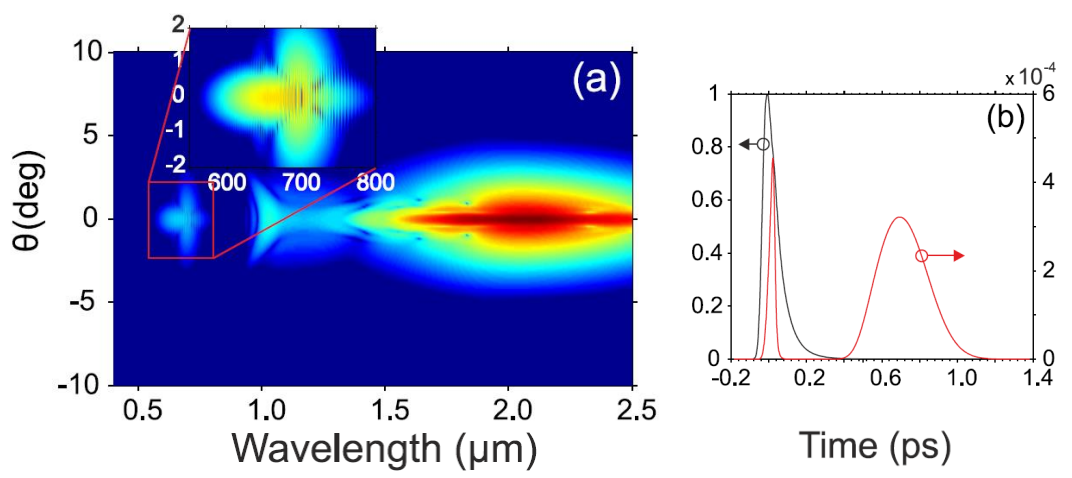

Fig. 11 (a) Spectrally resolved far-fields in YAG in the transient regime (a) of spectral broadening. The inset highlights the modulation around the third harmonic spectral range. (b) shows the normalized temporal profiles of the pump (black curve) and third harmonic (red curves) pulses after spectral filtering. Additional arrows in (b) indicate driven (on the left) and free (on the right) third harmonic pulses. Adapted from [5]

We interpret our results in the framework of large phase and group-velocity mismatched third harmonic generation [29]. The operating conditions imposes that the third harmonic radiation consists of two pulses, the free and driven waves, which are solutions to the homogenous and the inhomogenous wave equations, respectively. The free third harmonic wave propagates at the velocity $u_{f}$ set by the material dispersion, and walks-off from the pump pulse, i.e. the input-pulse at the fundamental frequency. The driven third harmonic wave propagates at the same velocity $u_{d}$ as the nonlinear polarization and appears as locked under the pump, i.e., travels at the pump pulse group velocity (see Figs. 10a,b). The emerging third harmonic radiation consists of two pulses separated in time by a duration $\left(u_{f}^{-1}-u_{d}^{-1}\right) L$, proportional tothe group velocity mismatch, where $L$ denotes the medium length. This produces beating in the third harmonic spectrum with frequency inversely proportional to the temporal separation between the free and driven components. By inserting the relevant values for YAG: $L=6 \mathrm{~mm}$ and group velocity mismatch $u=115 \mathrm{fs} / \mathrm{mm}$, the estimated temporal separation between the free and driven third harmonic pulses is $690 \mathrm{fs}$, which is very close to that of $670 \mathrm{fs}$, as retrieved from the fringe pattern shown in the spectrum.

In order to verify our interpretation, we performed numerical simulations with the model described in section 2. Figure 11a shows the result of numerical simulation for the spectrally resolved far-field in the transient regime of spectral broadening in YAG. The inset shows the magnified portion of the third harmonic spectrum in the 550-750 $\mathrm{nm}$ range, highlighting the spectral beatings. The profiles in Figure $11 \mathrm{~b}$ show the respective temporal profiles of the main (pump) pulse (black curves) and pulses at the third harmonic wavelength (red curves), retrieved by using a supergaussian filter with full width at half maximum of $150 \mathrm{~nm}$ and centered at $660 \mathrm{~nm}$. The retrieved third harmonic temporal profile consists of two distinct pulses temporally separated by 700 fs. They correspond to the free and driven third harmonic 
pulses, in fair agreement with our analytical interpretation and experimental results. The driven third harmonic pulse remains short as it is locked under the envelope of the fundamental (pump) pulse, which does not broaden much due to the interplay of self-phase modulation and anomalous dispersion, while the free third harmonic pulse moves away and experiences considerable temporal broadening due to normal dispersion.

\section{Conical light bullets generated by filamentation in the anomalous dispersion}

Figure 3 presented in introduction constitutes a typical result for filamentation in the anomalous dispersion regime of transparent solids. In an energy range above the threshold, the filament is very regular, featured by an absence of focusingdefocusing cycle sustaining filaments in the normal dispersion regime, at shorter wavelengths. These cycles, however, appear in the anomalous dispersion regime when the input pulse energy is increased and for slightly different focusing conditions [28] but after a few cycles, the filament regularizes and becomes regular as in Fig. 3 [6].

Numerical simulation results with the parameters of the experiments of Fig. 3 are shown in Fig. 12. The beam shrinks and forms the filament with a fairly constant diameter over more than $2 \mathrm{~cm}$ (Fig. 12a). Figure 12b shows the evolution of the axial temporal profile of the pulse, in the local frame, along the propagation distance. Both results show the emergence of a stable non spreading structure in space of time at the origin of the filamentary track extending over a large part of the sample. This structure possess some attributes of light-bullets, as introduced by Silberberg, refering to a pulse that propagates without changing its temporal or spatial shape, held together by the nonlinear forces [26]. Our measurements and simulations show quasi-stationary pulses, which do not change temporal or spatial shape for several diffraction lengths.

For instance, the duration of the pulse emerging from the sample was characterized by a single-shot, self-referenced spectral interferometry technique called Wizzler operating in the near infrared region [22]. The measurement shown in Fig. 13 corresponds to a a fused silica thickness of $5 \mathrm{~mm}$ and an integrated profile over 20 shots. A pulse duration of $20 \mathrm{fs}$ was obtained, with a fair reproducibility. Propagation of the infrared pulse has led to self-compression by a factor of two. However, the self-compressed structure do not qualify as light-bullets in Silberbergs sense for two reasons: (i) they continuously loose energy due to multiphoton absorption and (ii) they are stable whereas Silberberg's light bullets are unstable in more than one dimension.

In contracts, the pulse undergoinf filamentation in the anomalous dispersion regime was show in Ref. [20] to possess self-reconstruction properties and all the attributes of conical light bullets as proposed by Porras et al. [25]. The most important property of these structures is their conical nature allowing them to remain propa- 

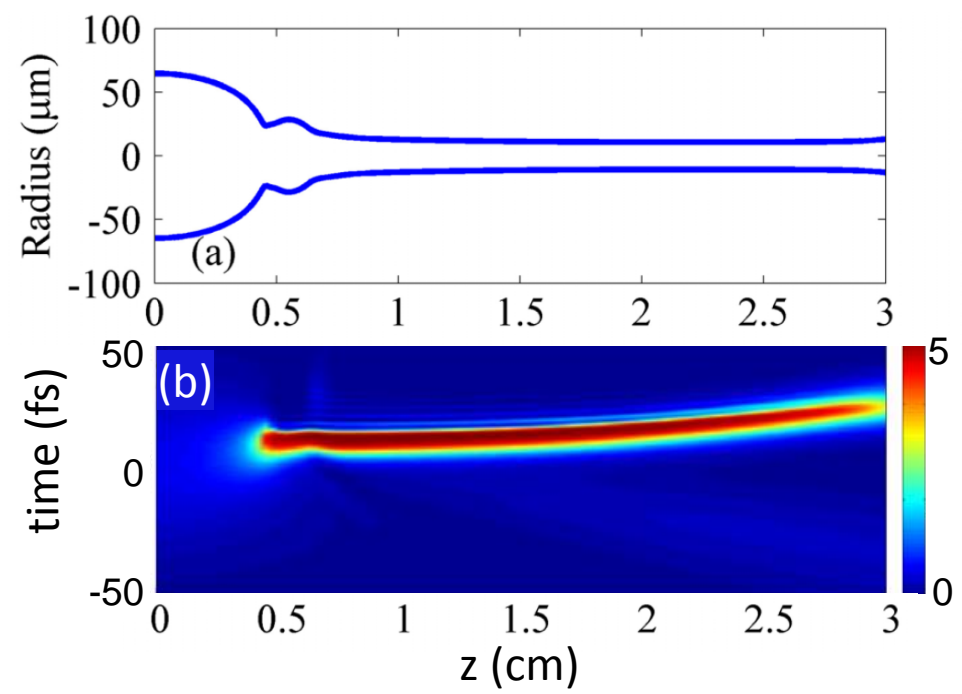

Fig. 12 Results of numerical simulation for the propagation through fused silica of an optical pulse at $1.9 \mu \mathrm{m}$ of $110 \mu \mathrm{m}$ diameter and $40 \mathrm{fs}$ duraton (FWHM) and $5 \times 10^{11} \mathrm{~W} / \mathrm{cm}^{2}$ intensity collimated on the front sample surface. (a) Beam radius as a function of distance $z$. Axial temporal profile of the laser pulse. Numbers on the colorbar denote TW/ $/ \mathrm{cm}^{2}$. Adapted from [6].

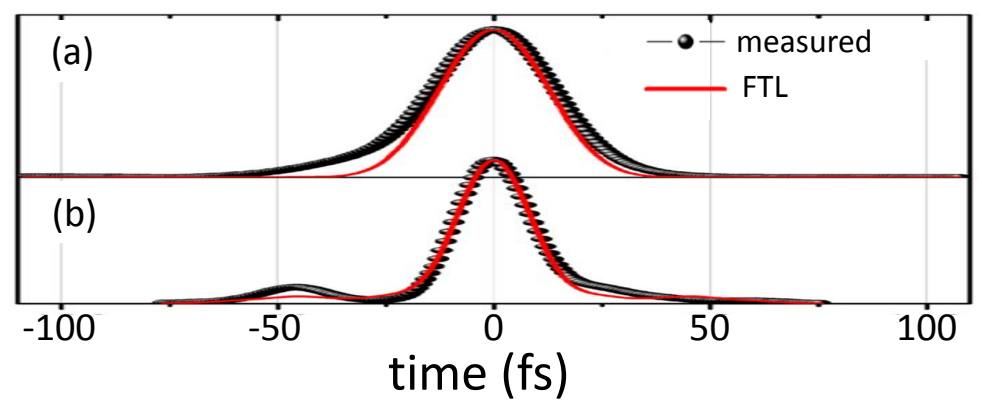

Fig. 13 Temporal pulse shape measured with the Wizzler for the initial pulse (40 fs FWHM) (a) and for filament lengths of $0.5 \mathrm{~cm}$ (20 fs FWHM) (b). Also shown in red is the Fourier Transform Limited (FTL) pulse. Adapted from [6].

gation invariant while undergoing multiphoton absorption. This is possible because the high intensity core where energy is lost is continually refueled by an energy flux from the low intensity energy reservoir of the wave. Invariant pulses in this form are found as mathematical solutions to a nonlinear Schrödinger equation accounting for the effects of diffraction, Kerr self-focusing, anomalous dispersion, and multiphoton absorption for any peak intensity up to a certain limit [25]. They are stable provided multiphoton absorption is sufficiently strong, and can therefore be observed in real experiments. 

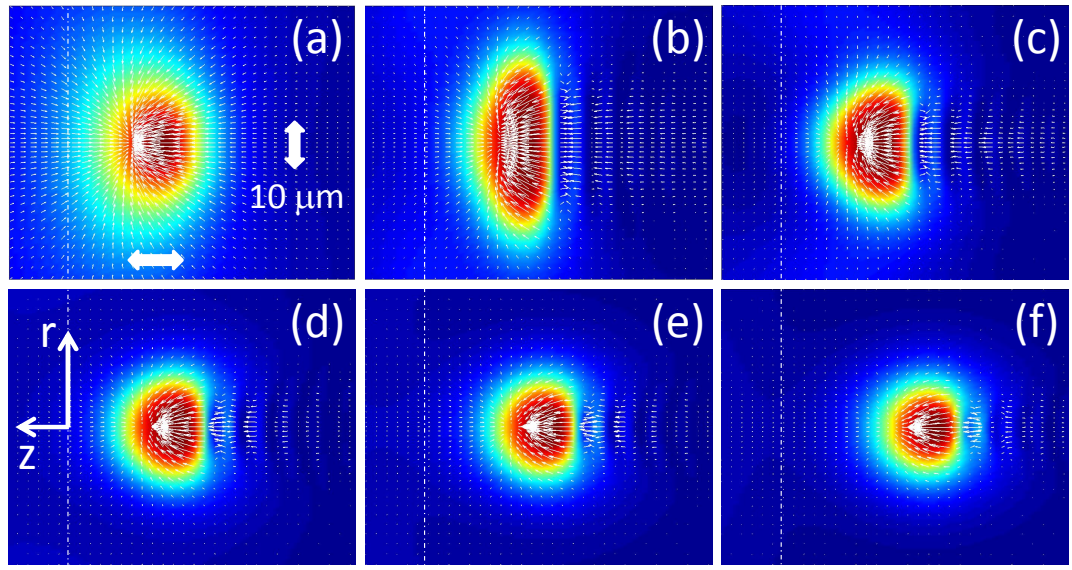

Fig. 14 Energy flux (white arrows) superimposed to intensity distribution (in color) obtained by numerical simulation at six propagation distances within a fused silica sample. (a) $z=4.5 \mathrm{~mm}$. (b) $z=6 \mathrm{~mm}$. (c) $z=7.5 \mathrm{~mm}$. (d) $z=12 \mathrm{~mm}$. (e) $z=15 \mathrm{~mm}$. (f) $z=19.5 \mathrm{~mm}$. The dash-dotted lines indicate the center of the input pulse (local frame center). The white arrows in (a) indicate the scale of $10 \mu \mathrm{m}$ valid for all figures. The axis in (f) indicate the transverse and the longitudinal coordinates $r, z$, with the longitudinal direction corresponding to the propagation direction.

A confirmation of the conical nature of the filament was obtained from numerical simulation results, showing the evolution of the energy density flux along the propagation in the fused silica sample. The complex field (intensity and phase) allows us to reconstruct the energy density flux in the full spatial and temporal coordinates [19]:

$$
\mathbf{F}=k_{0}^{-1} I\left[\left(\partial_{r} \phi\right) \mathbf{u}_{r}+\beta\left(\partial_{\zeta} \phi\right) \mathbf{u}_{z}\right],
$$

where $I(r, \zeta)$ and $\phi(r, \zeta)$ denote the intensity and phase distributions along transverse $r$ and longitudinal $\zeta \equiv k_{0}^{\prime-1} t-z$ axes, $\mathbf{u}_{r}$ and $\mathbf{u}_{\eta}$ are the unit vectors in the corresponding directions,

Figure 14 shows the energy flux components as arrows, along with an overlayed color plot of the intensity profiles at six distances along the propagation axis. Figure 14a-c evidence the pulse reshaping (compression in space and time) before the propagation invariant stage in Fig. 14d-f, sustained by a density flux essentially directed towards the leading and central part of the pulse from its periphery. A slow shift to the right corresponding to the subluminal motion of the pulse is the only visible change. The spatiotemporal distribution of the currents is almost identical to that of a conical light bullet [19]. We underline that Silberberg's bullets and solitons exhibit a flat phase and hence no transverse energy density flow in the spatiotemporal domain. Conversely, the energy density flux unveiled by the simulations is inherent to conical light bullets. Together with detailed measurements further highlighting the reconstruction properties of these bullets [20,14], these results unambiguously show the conical nature of the light bullets generated by self-focusing in a bulk dielectric medium with anomalous dispersion. 


\section{8 conclusion}

Laser pulse filamentation in transparent solids for near- and mid-infrared wavelengths lead to a significant spectral broadening and supercontinuum generation spanning more than three octaves. The pulse is efficiently self-compressed down to few-cycle pulse durations. Signatures in the form of free and driven third harmonic generation, conical emission and typical spectral peaks in the visible region, energy flux pointing toward the high intensity peak, propagation invariant $\mathrm{O}$-shaped spatiotemporal intensity profile and characteristic angularly resolved spectrum all point out established properties of conical light bullets.

Acknowledgements We acknowledge support from the French National Agency for Research, the French DGA, the ONERA, the European Social Fund under the Global Grant measure (Grant No. VP1-3.1-ŠMM-07-K-03-001), the Lithuanian Science Council, the Spanish Ministry of Education and Science through its Consolider Program Science (SAUUL CSD 2007-00013), through Plan Nacional (FIS2008-06368-C02-01), the Catalan Agencia de Gestio dAjuts Universitaris i de Recerca (AGAUR) with SGR 2009-2013, Fundacio Cellex Barcelona, and funding from LASERLAB-EUROPE, grant agreement 228334 and the Marie Curie Intra-European Fellowship program, the European Research Council under the European Unions Seventh Framework Programme (FP/2007-2013)/ERC GA 306559, the US Army Research Office MURI Program No. W911NF1110297, and the State of Florida.

\section{References}

1. M. Bass, C. DeCusatis, J. Enoch, V. Lakshminarayanan, G. Li, C. MacDonald, V. Mahajan, and E. Van Stryland. Handbook of Optics, 3rd Ed., volume 4. McGraw-Hill, 2009.

2. P. K. Bates, O. Chalus, and J. Biegert. Ultrashort pulse characterization in the mid-infrared. Opt. Lett., 35:1377-1379, 2010.

3. A. Couairon, E. Brambilla, T. Corti, D. Majus, O. de J. Ramírez-Góngora, and M. Kolesik. Practitioners guide to laser pulse propagation models and simulation. Eur. Phys. J. Special Topics, 199:5-76, 2011.

4. A. Couairon and A. Mysyrowicz. Femtosecond filamentation in transparent media. Physics reports, 441(2):47-189, 2007

5. J. Darginavičius, D. Majus, V. Jukna, N. Garejev, G. Valiulis, A. Couairon, and A. Dubietis. Ultrabroadband supercontinuum and third-harmonic generation in bulk solids with two optical-cycle carrier-envelope phase-stable pulses at $2 \mu \mathrm{m}$. Opt. Express, 21(21):25210, 2013.

6. M. Durand, A. Jarnac, A. Houard, Y. Liu, S. Grabielle, N. Forget, A. Durécu, A. Couairon, and A. Mysyrowicz. Self-guided propagation of ultrashort laser pulses in the anomalous dispersion region of transparent solids: A new regime of filamentation. Phys. Rev. Lett., 110:115003, 2013.

7. M. Durand, K. Lim, V. Jukna, E. McKee, M. Baudelet, A. Houard, M. Richardson, A. Mysyrowicz, and A. Couairon. Blueshifted continuum peaks from filamentation in the anomalous dispersion regime. Phys. Rev. A, 87:043820, 2013.

8. D. Faccio, A. Averchi, A. Couairon, A. Dubietis, R. Piskarskas, A. Matijosius, F. Bragheri, M. A. Porras, A. Piskarskas, and P. Di Trapani. Competition between phase-matching and stationarity in Kerr-driven optical pulse filamentation. Phys. Rev. E, 74:047603, 2006.

9. D. Faccio, A. Averchi, A. Lotti, P. Di Trapani, A. Couairon, D. Papazoglou, and S. Tzortzakis. Ultrashort laser pulse filamentation from spontaneous X-wave formation in air. Opt. Express, $16: 1565,2008$ 
10. D. Faccio, A. Matijosius, A. Dubietis, R. Piskarskas, A. Varanavičius, E. Gaižauskas, A. Piskarskas, A. Couairon, and P. Di Trapani. Near- and far-field evolution of laser pulse filaments in Kerr media. Phys. Rev. E, 72:037601, 2005.

11. D. Faccio, M. Porras, A. Dubietis, F. Bragheri, A. Couairon, and P. Di Trapani. Conical emission, pulse splitting and $\mathrm{X}$-wave parametric amplification in nonlinear dynamics of ultrashort light pulses. Phys. Rev. Lett., 96:193901, 2006.

12. D. Faccio, M. A. Porras, A. Dubietis, G. Tamošauskas, E. Kučinskas, A. Couairon, and P. Di Trapani. Angular and chromatic dispersion in Kerr-driven conical emission. Opt. Commun., 265:672-677, 2006.

13. N. Garejev, I. Gražulevičiūtė, D. Majus, G. Tamošauskas, V. Jukna, A. Couairon, and A. Dubietis. Third- and fifth-harmonic generation in transparent solids with few-optical-cycle midinfrared pulses. Phys. Rev. A, 89:033856, 2014.

14. I. Gražulevičiūtè, G. Tamošauskas, V. Jukna, A. Couairon, D. Faccio, and A. Dubietis. Selfreconstructing spatiotemporal light bullets. Optics Express, 22(25):30613, 2014.

15. M. Hemmer, M. Baudisch, A. Thai, A. Couairon, and J. Biegert. Self-compression to sub-3cycle duration of mid-infrared optical pulses in dielectrics. Opt. Express, 21:28095, 2013.

16. M. Kolesik and J. V. Moloney. Nonlinear optical pulse propagation simulation: From maxwell's to unidirectional equations. Phys. Rev. E, 70(3):036604, 2004.

17. M. Kolesik, E. M. Wright, and J. V. Moloney. Dynamic nonlinear X-waves for femtosecond pulse propagation in water. Phys. Rev. Lett., 92:253901, 2004.

18. M. Kolesik, E. M. Wright, and J. V. Moloney. Interpretation of the spectrally resolved far field of femtosecond pulses propagating in bulk nonlinear dispersive media. Opt. Express, 13(26):10729-10741, 2005.

19. A. Lotti, D. Faccio, A. Couairon, and P. Di Trapani. Energy-flux characterization of conical and space-time coupled wave packets. Phys. Rev. A, 81:023810, 2010.

20. D. Majus, G. Tamošauskas, I. Gražulevičiūtè, N. Garejev, A. Lotti, A. Couairon, D. Faccio, and A. Dubietis. Nature of spatiotemporal light bullets in bulk kerr media. Phys. Rev. Lett, 112:193901, 2014

21. K. D. Moll and A. L. Gaeta. Role of dispersion in multiple-collapse dynamics. Opt. Lett., 29(9):995-997, 2004.

22. T. Oksenhendler, S. Coudreau, N. Forget, V. Crozatier, S. Grabielle, R. Herzog, O. Gobert, and D. Kaplan. Self-referenced spectral interferometry. Appl. Phys. B, 99:7, 2010.

23. M. A. Porras, A. Dubietis, Ernestas Kučinskas, F. Bragheri, V. Degiorgio, A. Couairon, D. Faccio, and P. Di Trapani. From x- to o-shaped spatiotemporal spectra of light filaments in water. Opt. Lett., 30(24):3398-3400, 2005.

24. M. A. Porras, A. Dubietis, A. Matijošius, R. Piskarskas, F. Bragheri, A. Averchi, and P. Di Trapani. Characterization of conical emission of light filaments in media with anomalous dispersion. J. Opt. Soc. Am. B, 24(3):581, 2007.

25. M. A. Porras, A. Parola, and P. Di Trapani. Nonlinear unbalanced o waves: nonsolitary, conical light bullets in nonlinear dissipative media. J. Opt. Soc. Am. B, 22(7):1406, 2005.

26. Y. Silberberg. Collapse of optical pulses. Opt. Lett., 15(22):1282-1284, 1990.

27. F. Silva, D.R. Austin, A. Thai, M. Baudisch, M. Hemmer, D. Faccio, A. Couairon, and J. Biegert. Multi-octave supercontinuum generation from mid-infrared filamentation in a bulk crystal. Nat. Commun., 3:807, 2012.

28. E. O. Smetanina, V. O. Kompanets, S. V. Chekalin, A. E. Dormidonov, and V. P. Kandidov. Anti-Stokes wing of femtosecond laser filament supercontinuum in fused silica. Opt. Lett., 38:16, 2013.

29. G. Valiulis, V. Jukna, O. Jedrkiewicz, M. Clerici, E. Rubino, and P. Di Trapani. Propagation dynamics and X-pulse formation in phase-mismatched second-harmonic generation. Phys. Rev. A, 83:043834, 2011.

30. M. J. Weber. Handbook of Optical Materials. CRC Press NY, 2003. 